José Wémenson Rabelo

Chaves

wemensonrabelo@gmail.com Faculdade Cisne de Quixadá (FCQ)

Renan Calixto Pereira renancalixto@gmail.com Faculdade Cisne de Quixadá (FCQ)

Lucas Cavalcante de Almeida

lucascavalcante@det.ufc.br Centro Universitário Christus (Unichristus)

Francisco Heber Lacerda de Oliveira iD

heber@det.ufc.br

Universidade Federal do Ceará (UFC)

\section{Análise comparativa da irregularidade longitudinal de pavimento rodoviário em serviço por meio de aplicativos para smartphone}

\section{Comparative analysis of roughness of road pavement in service through smartphone applications}

\section{Análisis comparativo de la irregularidad longitudinal de suelo de carretera en servicio por medio de aplicaciones para Smartphone}

\section{Analyse comparative de l'irrégularité longitudinale de revêtement routier en service au moyen d'applications pour smartphone}

\section{Resumo}

A irregularidade longitudinal é um dos critérios mais usados na avaliação da qualidade funcional de um pavimento. Para realizar essa avaliação, os órgãos gestores de pavimentos têm a dificuldade de desenvolver ou escolher um modo preciso de obter esses dados, como também menos oneroso e de fácil operação. Diante desse contexto, o presente trabalho tem como objetivo avaliar a aplicabilidade dos aplicativos SmartIRI e RoadLab na avaliação de irregularidade longitudinal utilizando o Índice Internacional de Irregularidade (International Roughness Index - IRI). Avaliou-se, por meio dos aplicativos, a qualidade funcional dos trechos estudados, além de comparar os dados obtidos entre eles. Foi possível constatar que a utilização dos aplicativos pode ser uma alternativa viável para lugares em que há limitação de recursos financeiros, de equipamentos ou de pessoas capacitadas.

Palavras-chave: Avaliação funcional. Irregularidade longitudinal. SmartIRI. RoadLab.

\begin{abstract}
Rouoghness is one of the most used criteria for assessing the functional quality of a pavement. In order to carry out this assessment, the pavement management agencies have difficulty in developing a way to obtain this data in a precise manner, as well as, less costly and easy to operate. Given this context, the present paper aims to evaluate the applicability of the SmartIRI and RoadLab applications in the evaluation of roughness, through the International Roughness Index - IRI. Through the applications, the functional quality of the studied sections was evaluated, in addition to comparing the data obtained between them. It was found that the use of applications can be a viable alternative for places where there are limited financial resources, equipment or people trained for this purpose.
\end{abstract}

Keywords: Functional assessment. Roughness. SmartIRI. RoadLab.

\section{Resumen}

La irregularidad longitudinal es uno de los criterios más utilizados en la evaluación de la calidad funcional de un suelo. Para realizar esta evaluación, los órganos gestores de suelos tienen la dificultad de desarrollar o escoger un modo de obtener estos datos de manera precisa, como también, menos onerosa y de fácil operación. Ante este contexto, el presente trabajo 
tiene el objetivo de evaluar la aplicabilidad de las aplicaciones SmartIRI y RoadLab en la evaluación de irregularidad longitudinal, por medio del Índice Internacional de Irregularidad (International Roughness Index - IRI). A través de las aplicaciones se evaluó la calidad funcional de los tramos estudiados, además de comparar los datos obtenidos entre ellos. Fue posible constatar que la utilización de las aplicaciones puede ser una opción viable para lugares en que haya recursos financieros limitados, equipos o personas habilitadas para este fin.

Palabras-clave: Evaluación funcional. Irregularidad longitudinal. SmartIRI. RoadLab.

\section{Résumé}

L'irrégularité longitudinale est l'un des critères les plus utilisés dans l'évaluation de la qualité fonctionnelle d'un revêtement. Pour effectuer cette évaluation, les organismes de gestion de revêtement ont la difficulté de développer ou de choisir un moyen d'obtenir ces données avec précision, de façon moins coûteux et facile à utiliser. Dans ce contexte, ce travail vise à analyser l'utilisation des applications SmartIRI et RoadLab dans l'évaluation de l'irrégularité longitudinale, par le biais de l'Indice international d'irrégularité (IIR). Grâce aux applications, la qualité fonctionnelle des extraits a été évaluée. On a aussi pu comparer les données obtenues entre eux. II a été possible de vérifier que l'utilisation d'applications peut être une alternative viable où il y a des ressources financières, de l'équipement ou des personnes qualifiées limitées à cette fin.

Mots-clés : Évaluation fonctionnelle. Irrégularité longitudinale. SmartIRI. RoadLab.

\section{Introdução}

Um dos principais modos de transportes no Brasil é o rodoviário, sendo o mais importante para o deslocamento de cargas e de pessoas, responsável por $61 \%$ da matriz de carga e $95 \%$ da de passageiros. Assim, investir em infraestrutura de transporte é fundamental para fornecer segurança e conforto aos usuários, como também para favorecer o crescimento econômico do país, conforme afirma a pesquisa CNT (2018).

Ainda de acordo com a pesquisa CNT (2018), somente 12,4\% das rodovias brasileiras são pavimentadas, e, grande parte, apresentam deficiências, como buracos e remendos. Mediante o exposto, possivelmente, essas deficiências ocorrem por conta de manutenção do pavimento de modo insuficiente ou inadequado e do aumento da frota de veículos em circulação.

Os levantamentos e os dados obtidos durante a avaliação funcional dos pavimentos auxiliam na tomada de decisão sobre as melhorias e soluções de manutenção e reabilitação a serem executadas na infraestrutura rodoviária no decorrer do tempo. Um indicador comumente usado para tal avaliação é o Índice Internacional de Irregularidade (International Roughness Index - IRI), parâmetro estatístico, mensurado em m/km ou mm/m, que mede os desvios da superfície do pavimento em relação a uma superfície plana de referência. Todavia, para se obter informações sobre a irregularidade longitudinal e, assim, poder avaliar as condições funcionais do pavimento, existem várias alternativas, sendo uma grande parte delas, segundo Bernucci et al. (2010), bastante onerosa e demorada, além da necessidade da precisão dos valores obtidos.

Perante os problemas explanados e o avanço tecnológico, surgiram novos métodos e equipamentos de baixo custo que visam obter informações sobre as condições funcionais do pavimento, especialmente para cálculo do IRI. Segundo Almeida (2018), essas informações podem ser obtidas por meio de aplicativos para smartphones, tornando-se uma alternativa para estimar a condição da superfície do pavimento, proporcionando mais rapidez, menores custos e relativa precisão.

Diante do exposto, este trabalho tem como objetivo analisar o IRI de um pavimento rodoviário em serviço no município de Quixadá-CE, por meio de dados obtidos pelos aplicativos SmartIRI e RoadLab. Pretendeu-se, também, comparar a eficácia dessas tecnologias na avaliação funcional de pavimentos com revestimentos flexíveis e dos defeitos que possam contribuir para a majoração da irregularidade longitudinal nas vias. 


\section{Revisão bibliográfica}

Nesta seção, são apresentados conceitos sobre o IRI, os equipamentos medidores de irregularidade longitudinal e a sua classificação, além de conceitos acerca da aplicação de novas tecnologias, como a utilização de smartphones para medição do IRI e os aplicativos usuais existentes.

\section{1 Índice Internacional de Irregularidade (IRI)}

A qualidade do conforto ao rolamento durante o percurso nas estradas é uma grande preocupação dos órgãos gestores rodoviários, bem como para a maioria dos usuários que trafegam. Em razão disso, vários índices subjetivos foram desenvolvidos para estimar a serventia de uma via, como MPR (Mean Panel Rating), RN (Ride Number) e PSR (Present Serviceability Rating). No entanto essas avaliações subjetivas demandam tempo, profissionais treinados e alto valor financeiro. Como alternativa, desenvolveu-se um parâmetro designado Índice Internacional de Irregularidade (International Roughness Index - IRI), que mede a irregularidade longitudinal existente na superfície de uma estrada (YU; CHOU, YAU, 2006).

De acordo com o DNER (1994), a irregularidade longitudinal é o desvio de uma determinada superfície em relação a uma superfície plana de referência. Esses desvios influenciam a qualidade de rolamento, a dinâmica dos veículos sobre a via, a dinâmica das cargas e a drenagem do pavimento. Segundo Brown, Liu e Henning (2010), o IRI é um dos principais parâmetros para avaliação funcional do pavimento, que diz respeito ao conforto e à segurança que a rodovia fornece aos usuários, pois afeta a serventia, aumentando os custos operacionais dos veículos e a redução da vida útil do pavimento.

Segundo Duarte (2018), uma característica da irregularidade longitudinal existente em pavimentos é o fato que, a partir de um determinado valor inicial, ela cresce de forma exponencial, pois os desvios verticais da superfície influenciam na dinâmica das forças. A Figura 1 mostra os valores do IRI de acordo com o tipo e a condição funcional da superfície.

Figura 1 - Diversas faixas de variação do IRI dependendo do caso e da situação

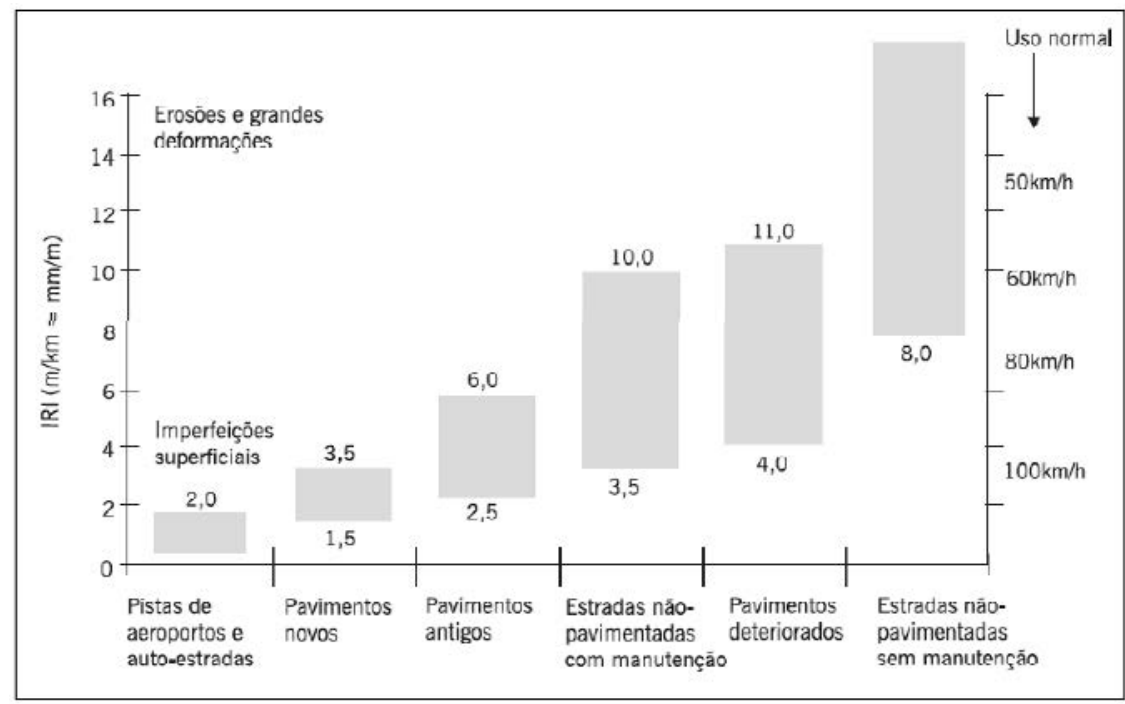

Fonte: Karamihas e Sayers (1998).

Comumente, a irregularidade longitudinal surge no decorrer de imperfeições no processo construtivo do pavimento, mas também pode resultar de adversidades após a execução, principalmente em decorrência da ação abrasiva do tráfego, intempéries e outros fatores (BUTTLAR; ISLAM, (2012). Segundo Almeida (2018), tem importância o estudo da irregularidade longitudinal em pavimentos rodoviários, uma vez que os 
resultados obtidos influenciam diretamente a tomada de decisão acerca dos investimentos para manutenção e reabilitação dessas vias.

\subsection{Equipamentos medidores de irregularidade longitudinal}

Existem diversos equipamentos usados para se medir a irregularidade longitudinal, obtendo-se, assim, o IRI. Segundo Bernucci et al. (2010), a irregularidade pode ser quantificada através de levantamentos topográficos ou mediante a utilização de equipamentos para medir o perfil longitudinal, com ou sem contato com a superfície, ou, ainda, indiretamente avaliada por equipamentos do tipo resposta, que fornecem um somatório de desvios do eixo de um veículo em relação a suspensão. Contudo, conforme Lerch, (2002), existe uma grande dificuldade dos equipamentos em expressar os desvios das variações longitudinais e transversais em um determinado índice numérico.

Ainda de acordo com Bernucci et al. (2010), os equipamentos medidores ou métodos utilizados para estimar a irregularidade longitudinal são divididos em quatro classes, tendo como base a capacidade de medir direta ou indiretamente as irregularidades em um determinado intervalo amostral. Um equipamento ser enquadrado em mais de uma classe, como se verifica na Tabela 1.

Tabela 1 - Classificação dos equipamentos medidores de irregularidade longitudinal

\begin{tabular}{|c|c|}
\hline Classe & Exemplos de Equipamentos ou Medição \\
\hline $\begin{array}{c}\text { Classe } 1 \\
\text { (Equipamentos de precisão que medem } \\
\text { diretamente o perfil dos pavimentos) }\end{array}$ & $\begin{array}{l}\text { - Nível e mira; } \\
\text { - Dipstick; } \\
\text { - Z-250; } \\
\text { - Perfilômetro do TRL; } \\
\text { - Perfilômetro Walking Profiler da ARRB. }\end{array}$ \\
\hline $\begin{array}{c}\text { Classe } 2 \\
\text { (Outros métodos perfilométricos) }\end{array}$ & $\begin{array}{l}\text { - Perfilógrafos; } \\
\text { - Equipamentos com sensores a laser; } \\
\text { - Infravermelho ou ultrassom; } \\
\text { - APL francês. }\end{array}$ \\
\hline $\begin{array}{c}\text { Classe } 3 \\
\text { (Sistemas medidores do tipo resposta) }\end{array}$ & $\begin{array}{l}\text { - Integrador de irregularidade longitudinal IPR/USP; } \\
\text { Maysmeter; } \\
\text { MERLIN; } \\
\text { Riley; } \\
\text { TRL Bump integrator. }\end{array}$ \\
\hline $\begin{array}{c}\text { Classe } 4 \\
\text { (Avaliações subjetivas) }\end{array}$ & - Painel de avaliadores. \\
\hline
\end{tabular}

Fonte: Gillespie et al. (1986).

\subsection{Utilização de smartphone na avaliação funcional dos pavimentos}

Segundo Bisconsini (2016), o uso de smartphones para mensurar a irregularidade longitudinal em pavimentos refere-se como um procedimento de medição do tipo resposta (classe III), ainda que não opere como um medidor tradicional da classe, que acumula os deslocamentos entre a carroceria e o eixo traseiro do automóvel, apesar de medir as acelerações verticais por meio de um smartphone fixado em um veículo. Segundo Tomiyama et al. (2012), a classificação desse tipo de dispositivo ocorre conforme a atribuição do sistema gerador de perfil e da sua adequação, conforme mostra a Figura 2. O "alvo" assinalado na Figura 2 refere-se a essa nova tecnologia, mostrando um sistema com melhor precisão e conveniência possível, porém ainda pertencente à classe dos sistemas medidores do tipo resposta. 
Figura 2 - Classificação dos smartphones em relação aos outros equipamentos

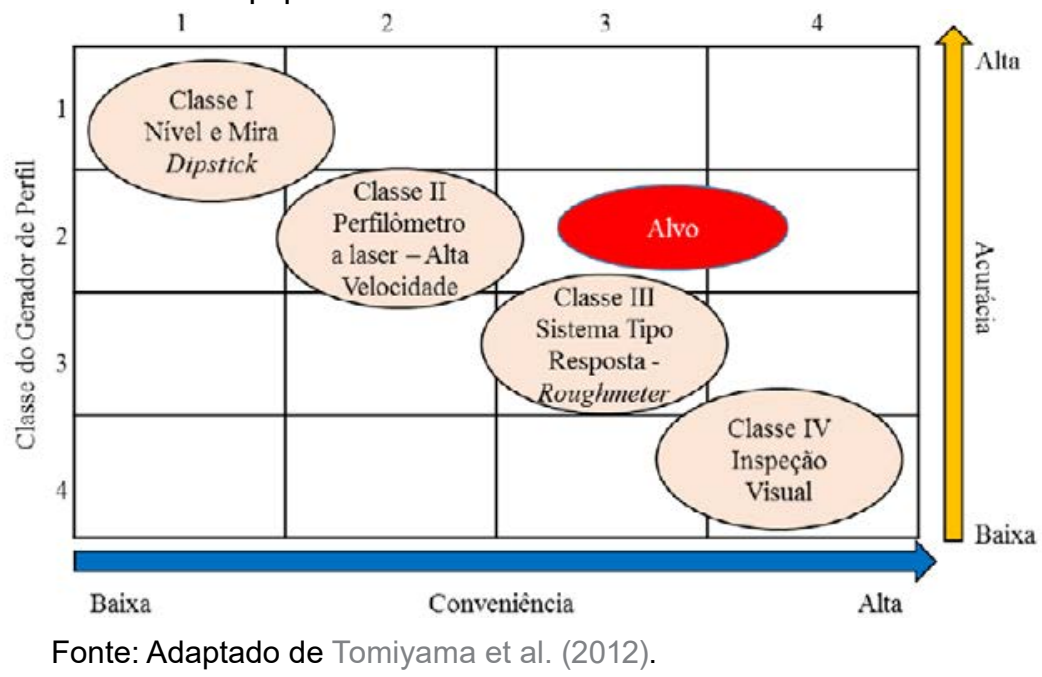

Em geral, a utilização de smartphones para estimar a irregularidade longitudinal tem mostrado resultados satisfatórios, com correlação positiva com os índices de irregularidade obtidos por outros equipamentos de medição. Com isso, mostram potencial para gerar informações importantes sobre a irregularidade, com nível de qualidade aceitável para um sistema de gerência de pavimentos (SCHLOTJES; VISSER; BENNET, 2014).

\section{4 aplicativos para smartphones disponíveis para medição da irregularidade longitudinal}

Segundo Del Rosario, Redmond e Lovell (2015), os avanços da indústria de smartphone possibilitaram o desenvolvimento de aparelhos integrados com as mais diversas funcionalidades e conectividades, como o sistema de posicionamento global (GPS) e sensores de movimentos (acelerômetro, magnetômetro e barômetro). Em razão disso, surgiu o interesse por utilizar esses sensores móveis para identificar e localizar as irregularidades e defeitos existentes nas vias, estimando a condição de serventia, principalmente por meio do parâmetro de IRI.

\subsubsection{RoadLab}

O aplicativo RoadLab foi desenvolvido por Wang e Guo (2016) com o propósito de informar as condições da rodovia acerca do conforto ao rolamento e da segurança. A ideia era que os próprios usuários que trafegam instalariam o RoadLab e forneceriam os dados acerca da infraestrutura para as agências gerenciadoras de pavimentos ou compartilhariam as informações coletadas com a comunidade online, por meio da divulgação dos locais com trechos críticos.

Para utilização do aplicativo é necessário que o smartphone seja fixado no painel ou para-brisa do veículo a ser utilizado, sendo a análise iniciada automaticamente quando o automóvel atingir uma velocidade de $30 \mathrm{~km} / \mathrm{h}$. Os valores de IRI são coletados por meio da aceleração, captada pelo sensor de movimento (acelerômetro) e georreferenciado pelo GPS do smartphone. A partir desses dados, verificam-se os picos de aceleração ocorridos durante o percurso, evidenciando os locais com irregularidade (WANG; GUO, (2016).

Os desenvolvedores do RoadLab realizaram a calibragem por regressão para aferir os valores de IRI do aplicativo, possuindo como base os valores de desvio padrão da aceleração vertical e valores de velocidade operacional do veículo em trechos com as mais variadas condições de irregularidade, específicas do país desenvolvedor (DUARTE, 2018).

\subsubsection{SmartIRI}

O SmartIRI é um aplicativo para Android desenvolvido por Almeida (2018), na Universidade Federal do Ceará (UFC), para a avaliação das rodovias brasileiras, em especial as vias do estado do Ceará, calculando o 
IRI através de correlação com o RMSVA (Root Mean Square Vertical Acceleration). O próprio aplicativo realiza o tratamento dos dados, fornecendo os parâmetros de IRI, isto é, a hora da avaliação, a velocidade média, a distância percorrida e as coordenadas geográficas do trecho analisado. Segundo Almeida (2018), o intuito foi desenvolver um aplicativo de fácil manuseio e, ao mesmo tempo, eficiente em questão de gerenciamento do consumo de energia do smartphone, não afetando a vida útil da bateria, assim como a performance do dispositivo durante o funcionamento do aplicativo. A respeito da precisão dos resultados, os levantamentos efetuados por Almeida (2018) expuseram que os valores obtidos pelo SmartIRI foram condizente com outros métodos ou classes de equipamentos na maioria dos testes, como o VSA, o perfilômetro a laser e o nível e mira de precisão.

Para sua utilização, o smartphone, com o SmartIR/ instalado, necessita de prévia calibração na posição vertical. Após a calibração, o aparelho deve ser fixado no para-brisa do veículo por meio de um suporte e a avaliação deve ser iniciada quando o veículo estiver em movimento, com velocidade de 60 a $80 \mathrm{~km} / \mathrm{h}$ (ALMEIDA, 2018). A Tabela 2 mostra o sistema de classificação adotado pelo aplicativo.

Tabela 2 - Classificação proposta pelo SmartIRI e RoadLab

\begin{tabular}{l|l}
\hline Intervalos de IRI $(\mathbf{m} / \mathbf{K m})$ & Classificação \\
\hline $\mid \mathrm{RI}<2$ & Excelente \\
$2 \leq \mid \mathrm{RI}<4$ & Bom \\
$4 \leq \mathrm{IRI}<6$ & Regular \\
$6 \leq \mathrm{IRI}$ & Ruim \\
\hline
\end{tabular}

Fonte: Adaptado de Almeida (2018).

\section{Método de pesquisa}

Nesta seção, é realizada uma descrição do trecho da rodovia federal estudada, além de demonstrar o procedimento para a obtenção dos dados e como os resultados obtidos foram estruturados de modo que fosse possível analisá-los de forma organizada e coerente.

\subsection{Descrição do trecho analisado}

Para este estudo, analisou-se um trecho da rodovia federal BR-122. O trecho selecionado está localizado no município de Quixadá, conhecido como estrada do Algodão, com extensão de, aproximadamente, 1,5 km, possuindo uma pista simples de rolamento e duas faixas de tráfego (uma em cada sentido), e pavimentação em revestimento flexível. A Figura 3 mostra o trecho, e os pontos com indicações A e B designam, respectivamente, o início e o final do levantamento para as faixas de tráfego (F1 e F2)

Figura 3 - Trecho avaliado com extensão de $1,5 \mathrm{~km}$ e faixas de tráfego
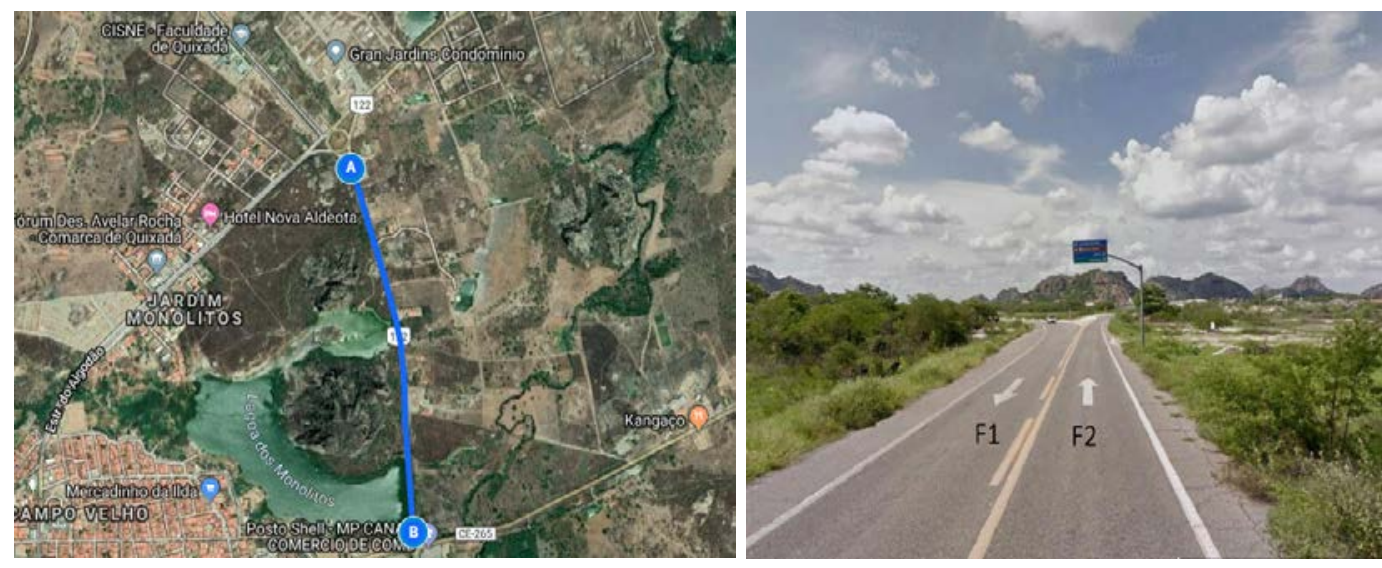

Fonte: Google Earth (2019).

Fonte: Os autores (2019). 


\subsection{Levantamento e análise de dados}

A coleta de dados foi realizada em agosto de 2019, em condições climáticas satisfatórias, sem ocorrências de precipitações que possam prejudicar a visibilidade do trecho, ressaltando que tal condição não é um fator de análise dos aplicativos. Para o levantamento, utilizou-se o smartphone Galaxy, da marca Samsung, contendo as funcionalidades necessárias para medição, que são: giroscópio, acelerômetro e GPS, para a georreferenciação do trecho. O aparelho foi fixado no para-brisa de um veículo da marca Hyundai, modelo HB20, motorização 1.6 e ano 2016, com todas as funções operacionais revisadas e pneus calibrados em 32 psi, de acordo com a orientação do fabricante, com velocidade média de $60 \mathrm{~km} / \mathrm{h}$, pois é a velocidade máxima permitida na vida.

Quanto ao suporte veicular para smartphones empregado, já que se trata de uma medição do tipo resposta, ou seja, que depende da resposta do pavimento com a suspensão do carro, as vibrações excessivas e desnecessárias tirariam a acurácia nos resultados obtidos, dessa maneira, utilizou-se o modelo Vehicle Dock da marca Samsung, como recomendado por Forslöf e Jones (2013). O suporte recomendado mostrouse eficiente diante do problema relatado e contribuiu para atenuar erros causados pela vibração excessiva, como mostra a Figura 4.

Figura 4 - Aplicativo SmartIRI em uso no trecho analisando e o suporte mencionado

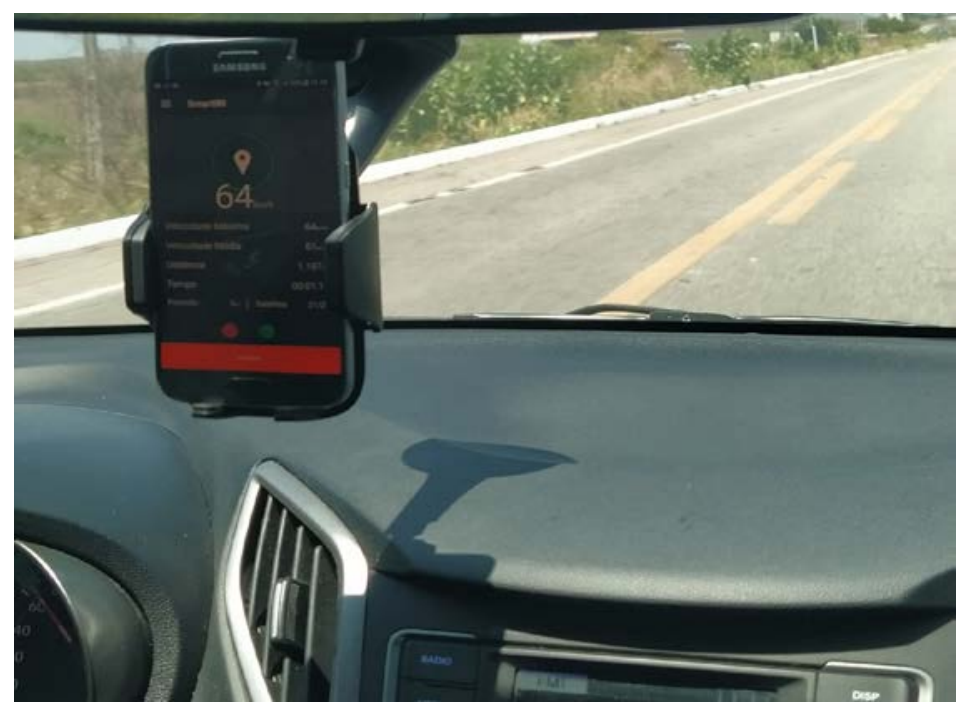

Fonte: Os autores (2019).

Os levantamentos foram realizados em cada sentido da faixa de tráfego, sendo uma vez com o aplicativo SmartIRI e duas vezes com o aplicativo RoadLab, pois este último não conseguiu mensurar os valores do IRI em alguns trechos da via devido a falhas do aplicativo ao acessar os dados de GPS. Logo, foram realizadas duas passagens e feita a média aritmética dos valores coletados, descartando os que não foram detectados pelo RoadLab. Durante o levantamento, evitou-se desviar de defeitos presentes no pavimento, de forma a coletar resultados coerentes com a situação real existente na superfície do trecho.

Após o término do levantamento de dados em cada faixa de tráfego, os próprios aplicativos forneceram uma planilha com os resultados obtidos, em intervalos de leitura de aproximadamente 100 metros, classificados em uma escala qualitativa e quantitativa dos valores de IRI. De posse da planilha eletrônica com os valores de IRI e os trechos georreferenciados, realizou-se uma análise descritiva desses dados, por meio do software Excel, gerando gráficos e parâmetros estatísticos. 


\section{Resultados e discussão}

Nesta seção, são discutidos e comparados os valores de IRI obtidos no trecho avaliado por meio dos aplicativos SmartIRI e RoadLab, além da análise acerca dos defeitos existentes no pavimento da área de estudo.

\subsection{Análise dos dados}

As Tabelas 3 e 4 mostram os valores de IRI gerados pelo SmartIRI para as duas faixas de tráfego. Analisando-se os dados obtidos, verifica-se que $25 \%$ dos segmentos da Faixa 1 e $16,67 \%$ da Faixa 2 foram classificados com os conceitos de IRI regular e ruim.

Tabela 3 - IRI obtidos pelo aplicativo SmartIRI para a faixa de tráfego 1

\begin{tabular}{c|c|c|c|cl}
\hline Faixa & Segmento & $\begin{array}{c}\text { Velocidade } \\
\text { média }(\mathbf{k m} / \mathbf{h})\end{array}$ & $\begin{array}{c}\text { Distância } \\
\text { acumulada }(\mathbf{m})\end{array}$ & $\begin{array}{c}\text { IRI } \\
\mathbf{( m / \mathbf { m }} \mathbf{)}\end{array}$ & Classificação \\
\hline \multirow{6}{*}{ F1 } & 59 & 116,00 & 4,26 & Regular \\
& 1 & 59 & 229,00 & 3,77 & Bom \\
& 2 & 60 & 329,00 & 3,48 & Bom \\
& 3 & 61 & 430,00 & 4,39 & Regular \\
& 5 & 60 & 546,00 & 3,53 & Bom \\
& 6 & 59 & 660,00 & 6,80 & Ruim \\
& 7 & 62 & 762,00 & 3,75 & Bom \\
& 8 & 62 & 865,00 & 3,90 & Bom \\
& 9 & 60 & 965,00 & 2,82 & Bom \\
& 10 & 63 & 1069,00 & 3,01 & Bom \\
& 11 & 63 & 1174,00 & 3,40 & Bom \\
\hline
\end{tabular}

Fonte: Os autores (2019).

Tabela 4 - IRI obtidos pelo aplicativo SmartIRI para a faixa de tráfego 2

\begin{tabular}{c|c|c|c|cl}
\hline Faixa & Segmento & $\begin{array}{c}\text { Velocidade } \\
\text { média }(\mathbf{k m} / \mathbf{h})\end{array}$ & $\begin{array}{c}\text { Distância } \\
\text { acumulada }(\mathbf{m})\end{array}$ & $\begin{array}{c}\text { IRI } \\
(\mathbf{m} / \mathbf{k m})\end{array}$ & Classificação \\
\hline \multirow{6}{*}{ F2 } & 1 & 65 & 108,00 & 2,60 & Bom \\
& 2 & 63 & 213,00 & 3,10 & Bom \\
& 3 & 62 & 316,00 & 2,80 & Bom \\
& 4 & 61 & 418,00 & 3,59 & Bom \\
& 5 & 64 & 525,00 & 3,12 & Bom \\
& 6 & 65 & 633,00 & 4,43 & Regular \\
& 7 & 64 & 739,00 & 3,12 & Bom \\
& 8 & 63 & 843,00 & 3,86 & Bom \\
& 9 & 60 & 943,00 & 3,49 & Bom \\
& 10 & 59 & 1058,00 & 4,17 & Regular \\
& 11 & 59 & 1172,00 & 3,21 & Bom \\
\hline
\end{tabular}

Fonte: Os autores (2019).

As Tabelas 5 e 6 apresentam os resultados obtidos pelo aplicativo RoadLab nas duas passagens. Analisando-se as Tabela 5 e 6, nota-se que a grande maioria dos segmentos na Faixa 1 recebeu o conceito regular (85,71\%), enquanto, na Faixa 2, a maioria dos segmentos receberam o conceito bom $(64,29 \%)$. 
Tabela 5 - IRI obtidos pelo aplicativo RoadLab para as faixas de tráfego F1

\begin{tabular}{c|c|c|c|c|l}
\hline Faixa & Segmento & $\begin{array}{c}\text { Velocidade } \\
\text { média } \mathbf{( k m} / \mathbf{h})\end{array}$ & $\begin{array}{c}\text { Distância } \\
\text { acumulada } \mathbf{( m )}\end{array}$ & $\begin{array}{c}\text { IRI } \\
\mathbf{( m / k m} \mathbf{~})\end{array}$ & Classificação \\
\hline \multirow{6}{*}{ F1 } & 1 & 50,72 & 113,34 & 5,08 & Regular \\
& 2 & 55,79 & 222,80 & 5,94 & Regular \\
& 3 & 57,50 & 334,46 & 4,83 & Regular \\
& 4 & 57,26 & 445,91 & 4,57 & Regular \\
& 5 & 58,63 & 561,17 & 4,05 & Regular \\
& 6 & 60,40 & 662,38 & 4,54 & Regular \\
& 7 & 59,50 & 777,82 & 4,64 & Regular \\
& 8 & 59,24 & 893,08 & 4,55 & Regular \\
& 9 & 61,25 & 995,18 & 5,15 & Regular \\
& 10 & 59,74 & 1095,31 & 4,15 & Regular \\
& 11 & 57,75 & 1207,99 & 3,77 & Bom \\
& 12 & 63,00 & 1314,19 & 4,03 & Regular \\
& 13 & 62,98 & 1418,23 & 4,15 & Regular \\
\hline
\end{tabular}

Fonte: Os autores (2019).

Tabela 6 - IRI obtidos pelo aplicativo RoadLab para as faixas de tráfego F2

\begin{tabular}{|c|c|c|c|c|c|}
\hline Faixa & Segmento & $\begin{array}{l}\text { Velocidade média } \\
(\mathrm{km} / \mathrm{h})\end{array}$ & $\begin{array}{c}\text { Distância acumu- } \\
\text { lada (m) }\end{array}$ & $\begin{array}{c}\text { IRI } \\
(\mathrm{m} / \mathrm{km})\end{array}$ & Classificação \\
\hline \multirow{14}{*}{$\mathbf{F 2}$} & 1 & 50,56 & 103,60 & 1,07 & Excelente \\
\hline & 2 & 59,88 & 204,30 & 3,61 & Bom \\
\hline & 3 & 60,88 & 305,30 & 2,94 & Bom \\
\hline & 4 & 59,91 & 405,60 & 3,11 & Bom \\
\hline & 5 & 58,72 & 519,50 & 3,25 & Bom \\
\hline & 6 & 61,89 & 623,10 & 4,36 & Regular \\
\hline & 7 & 64,70 & 730,60 & 3,51 & Bom \\
\hline & 8 & 63,53 & 835,80 & 4,20 & Regular \\
\hline & 9 & 61,99 & 938,80 & 5,00 & Regular \\
\hline & 10 & 60,89 & 1040,10 & 4,20 & Regular \\
\hline & 11 & 61,48 & 1142,50 & 3,75 & Bom \\
\hline & 12 & 62,75 & 1246,90 & 2,95 & Bom \\
\hline & 13 & 61,68 & 1348,70 & 3,82 & Bom \\
\hline & 14 & 61,00 & 1449,60 & 3,63 & Bom \\
\hline
\end{tabular}

Fonte: Os autores (2019).

Comparando-se de forma qualitativa as classes (excelente, bom, regular e ruim) dos valores de IRI obtidos por meio dos aplicativos SmartIRI e RoadLab para a classificação dos segmentos, como mostrado na Tabela 7, observa-se que a classificação da Faixa F2 foi condizente nos dois aplicativos, pois a maioria dos segmentos tiveram atribuídos valores com conceito bom. Contudo a Faixa F1 apresentou uma divergência de classificação entre os aplicativos, posto que, no SmartIRI, grande parte dos segmentos é classificado com o conceito bom, enquanto, no RoadLab, apresentou o conceito regular. Apesar do número de trechos medidos pelos aplicativos serem diferentes, ressalta-se que a diferença entre a quantidade e extensão dos trechos medidos é cerca de 70 metros. Tal imprecisão é resultante da coleta realizada via GPS, que pode ocasionar variação nos valores das coordenadas com o veículo em movimento. 
José Wémenson Rabelo Chaves, Renan Calixto Pereira, Lucas Cavalcante de Almeida, Francisco Heber Lacerda de Oliveira

Tabela 7 - Resumo dos resultados obtidos por meio dos aplicativos RoadLab e SmartIRI

\begin{tabular}{l|c|c|c|c}
\hline Classificação & \multicolumn{2}{|c|}{ SmartIRI } & \multicolumn{2}{c}{ RoadLab } \\
\hline Faixa & $\mathrm{F} 1$ & $\mathrm{~F} 2$ & $\mathrm{~F} 1$ & $\mathrm{~F} 2$ \\
\hline Excelente & $0,00 \%$ & $0,00 \%$ & $0,00 \%$ & $7,14 \%$ \\
Bom & $75,00 \%$ & $83,33 \%$ & $14,29 \%$ & $64,29 \%$ \\
Regular & $16,67 \%$ & $16,67 \%$ & $85,71 \%$ & $28,57 \%$ \\
Ruim & $8,33 \%$ & $0,00 \%$ & $0,00 \%$ & $0,00 \%$ \\
\hline
\end{tabular}

Fonte: Os autores (2019).

Essa divergência de valores e classificação entre os aplicativos pode ser ocasionada devido a forma de calibração dos desenvolvedores, já que o SmartIRI foi desenvolvido e calibrado para as condições de rodovias brasileiras, particularmente as rodovias do estado do Ceará, enquanto que o Roadlab tem sua aplicação em países estrangeiros, possuindo um espectro de medição, utilizado na calibração do aplicativo, possivelmente inadequado à variação da irregularidade para a maioria das rodovias brasileiras.

Por meio de análise estatística dos dados coletados, verificou-se que o coeficiente de variação para a Faixa 1 foi de $27,27 \%$ no SmartIRI e $15,88 \%$ no RoadLab. Observou-se, na média geral dos valores de IRI obtidos por faixa, que os aplicativos obtiveram valores aproximados na Faixa 2, conforme mostra a Tabela 8.

Tabela 8 - Análise estatística descritiva dos trechos analisados

\begin{tabular}{l|c|c|c|c}
\hline Análise estatística & \multicolumn{2}{|c|}{ SmartIRI } & \multicolumn{2}{c}{ RoadLab } \\
\hline Trecho & $\mathrm{F} 1$ & $\mathrm{~F} 2$ & $\mathrm{~F} 1$ & $\mathrm{~F} 2$ \\
Média & 3,85 & 3,40 & 0,45 & 3,52 \\
Desvio padrão & 1,05 & 0,54 & 0,73 & 0,91 \\
Coeficiente de variação & $27,27 \%$ & $15,88 \%$ & $16,40 \%$ & $25,94 \%$ \\
Maior valor & 6,80 & 4,43 & 5,93 & 1,07 \\
Menor valor & 2,82 & 2,60 & 2,83 & 5,00 \\
\hline Classificação & Bom & Bom & Regular & Bom \\
\hline
\end{tabular}

Fonte: Os autores (2019).

De maneira a verificar se existe diferença significativa entre as médias gerais obtidas por faixa pelos dois aplicativos, realizou-se um teste t pareado. Adotando a hipótese nula como a igualdade entre as médias, ou seja, sua diferença é igual a zero, e hipótese alternativa que as médias são diferentes. Por meio do teste, verificou-se que, para as médias de IRI na Faixa 1 e na Faixa 2, o P-Valor possui um valor maior que o nível de significância $(0,05)$ (Tabela 9), sendo, portanto, a hipótese nula aceita, significando que não existe uma diferença significativa entre as médias gerais.

Tabela 9 - Resultado do teste t pareado

\begin{tabular}{c|c}
\hline Faixas & P-valor \\
\hline F1 & 0,075 \\
F2 & 0,483 \\
\hline
\end{tabular}

Fonte: Os autores (2019).

Analisando os gráficos das Figuras 5 e 6, notou-se que os aplicativos SmartIRI e RoadLab apresentaram variações dos valores de IRI ao longo dos dois trechos, apresentando divergência de distribuição dos dados levantados para as faixas tráfego, ocorrendo frequentes picos de valores de IRI. Ressalta-se que o aplicativo RoadLab perdeu ou deixou de coletar algumas medições, provavelmente por ausência de sinal do GPS ou vibrações excessivas, o que não ocorreu com o SmartIRI. 
Figura 5 - Valores de IRI versus distância acumulada na Faixa

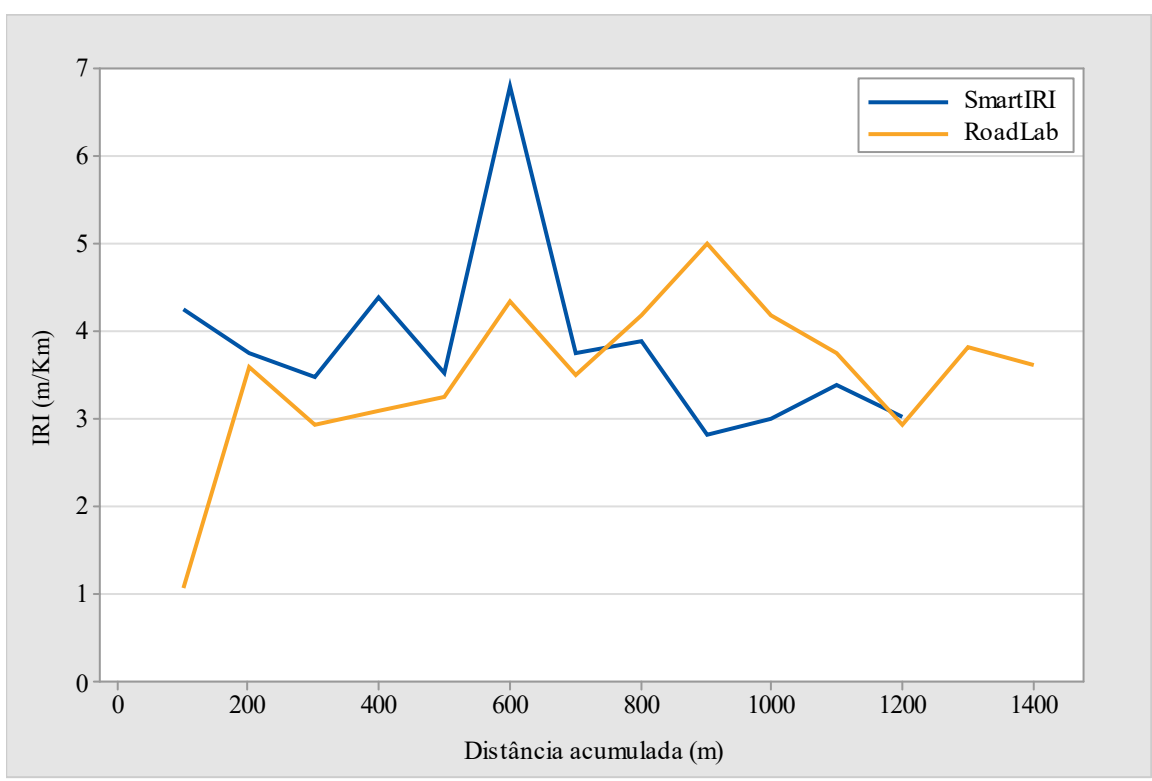

Fonte: Os autores (2019).

Figura 6 - Valores de IRI versus distância acumulada na Faixa 2

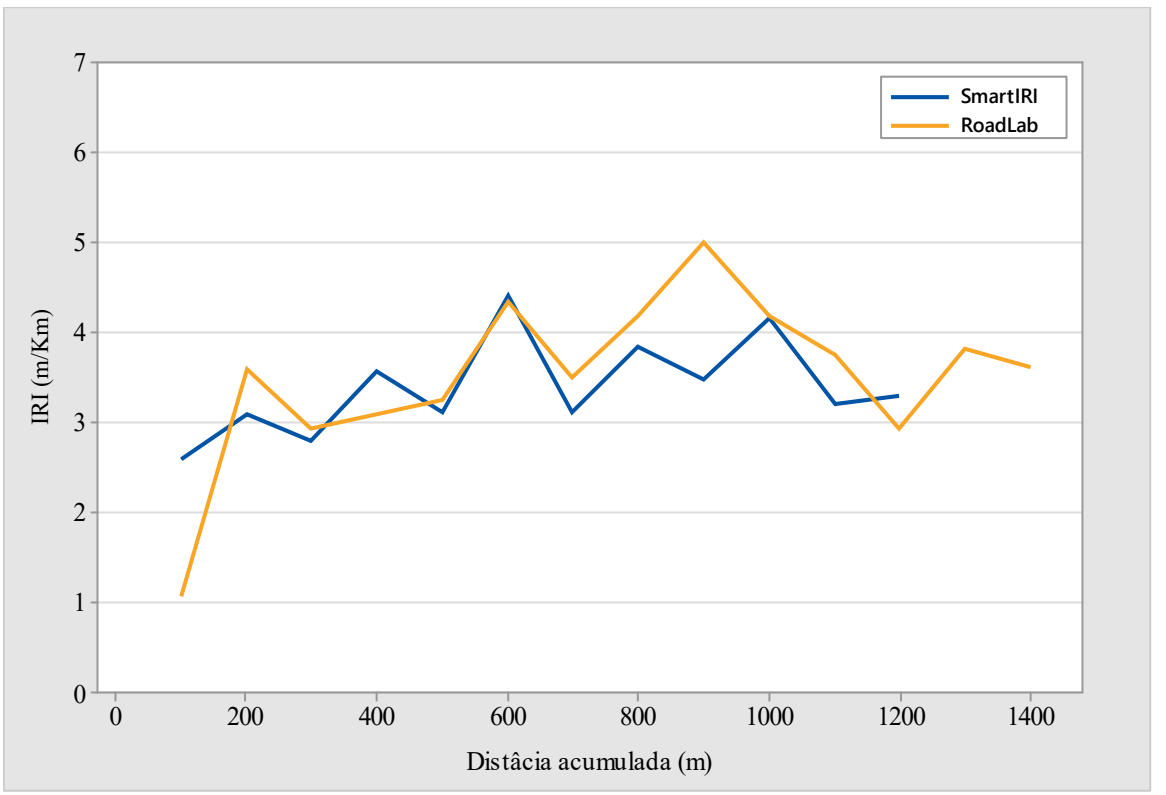

Fonte: Os autores (2019).

Em alguns locais do trecho avaliado, observaram-se defeitos que podem ter contribuído para a majoração do IRI, quais sejam: o remendo, os buracos e as trincas já com alta severidade. Embora a textura aberta do pavimento não seja considerada como um defeito, essa característica tende a contribuir para o acréscimo dos valores de IRI. Constatou-se que um buraco presente na Faixa 2, especificamente no segmento 6 do trecho estudado (Figura 7), pode ter influenciado a obtenção do valor de IRI acima dos demais segmentos. 
Figura 7 - Defeito do tipo buraco localizado na Faixa 2 do trecho estudado

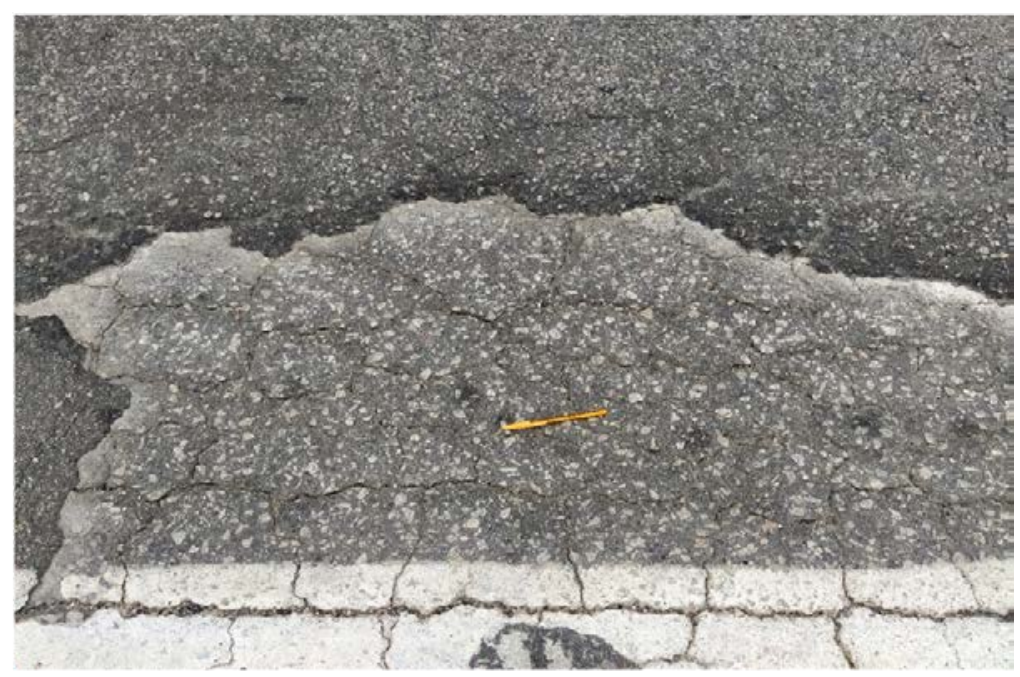

Fonte: Os Autores (2019).

Apesar das trincas não interferirem no aumento do IRI da rodovia, a textura aberta pode contribuir para o aumento da irregularidade longitudinal, conforme afirmam Almeida, Oliveira e Ramos (2018). Observaram-se que, em grande parte da via inspecionada, existem defeitos dos tipos trinca e desgaste da superfície (Figura 8), além da presença de textura aberta.

Figura 8 - Trinca e desgastes da superfície da via estudada

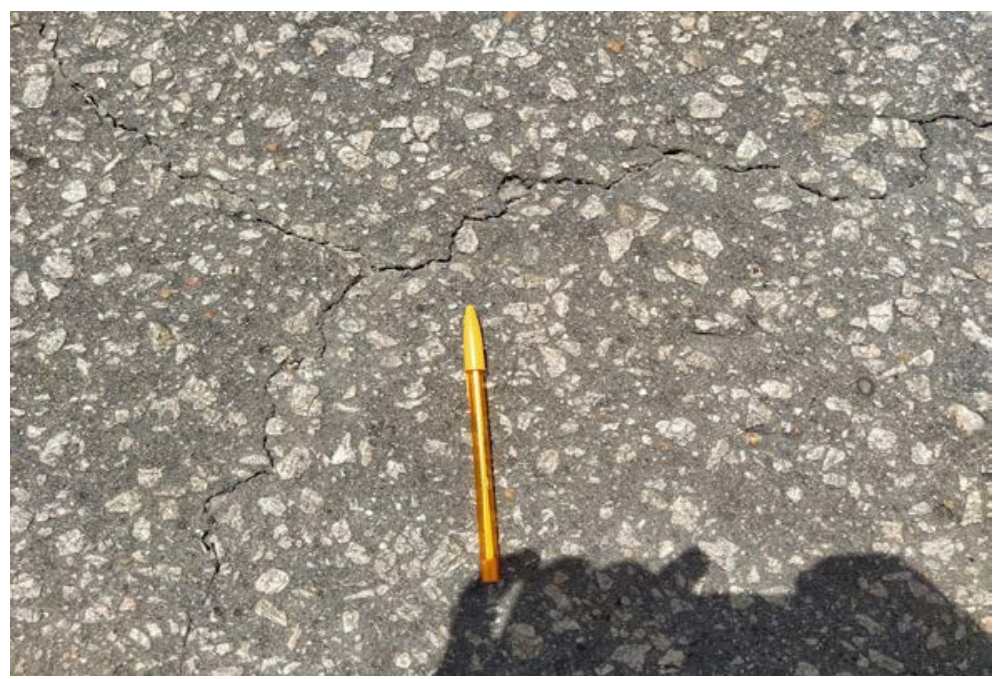

Fonte: Os Autores (2019).

Por sua vez, a Figura 9 mostra o principal defeito encontrado no trecho, que foram os remendos, aumentando o IRI da Faixa 2, no segmento 9, e, consequentemente, afetando a qualidade de rolamento da rodovia. 
Figura 9 - Remendos encontrados no pavimento inspecionado

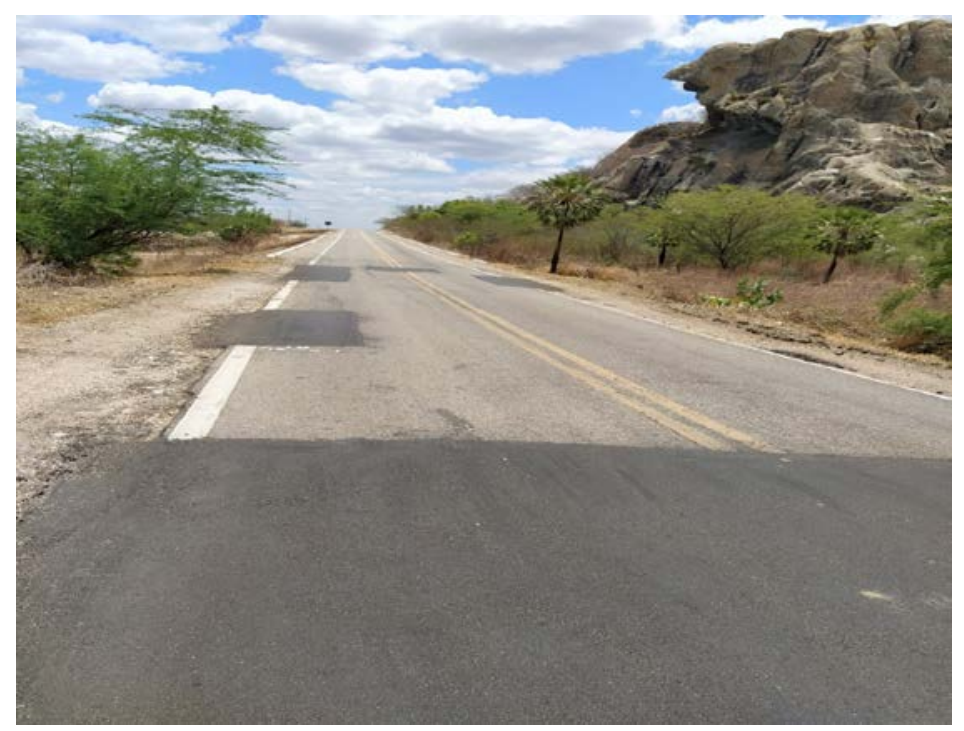

Fonte: Os Autores (2019).

\section{Conclusão}

Este trabalho analisou o IRI de um trecho rodoviário em pavimento flexível por meio dos aplicativos para smartphone SmartIRI e RoadLab. Procurou-se utilizar esse meio de avaliação por ser um equipamento de baixo custo, alta produtividade e de fácil manuseio na operação. Os aplicativos mostraram-se eficientes em termos de simplicidade na coleta dos dados. Quanto à acurácia, não foi feita a comparação com métodos de levantamento já consagrados no meio rodoviário.

O aplicativo SmartIRI, por ser um aplicativo nacional, obteve vantagem na simplicidade e objetividade da sua operação, além de não ter acontecido falhas durante seu uso, mensurando todo o trecho especificado. Já o aplicativo RoadLab apresentou algumas falhas durante o seu uso, deixando de mensurar algumas partes do trecho.

No trecho, foram encontrados alguns defeitos que afetam a qualidade do rolamento na via, sendo os principais buracos e remendos, além da textura do pavimento, que é aberta, afetando a qualidade durante o rolamento. Foi possível identificar algumas trincas no trecho relatado. Esse defeito, em um estágio avançado, pode afetar a qualidade do rolamento.

Concluiu-se que os aplicativos para smartphones mostram-se uma boa alternativa em relação a tempo e disponibilidade dos dados, podendo facilitar a tomada de decisão dos órgãos gestores brasileiros, no entanto ainda carecem de mais comparações com equipamentos ou métodos já normatizados por órgãos rodoviários.

\section{Referências}

ALMEIDA, L. C. Aplicativo para smartphone destinado à medição da irregularidade longitudinal em rodovias. 2018. 93 f. Dissertação (Mestrado em Engenharia de Transportes) - Universidade Federal do Ceará, Fortaleza, 2018. Disponível em: http://www.repositorio.ufc.br/bitstream/riufc/35669/3/2018_dis_ Icalmeida.pdf. Acesso em: 20 abr. 2019.

ALMEIDA, L. C; OLIVEIRA, F. H. L; RAMOS, S. P. Estudo da condição de superfície em rodovias por meio do uso de aplicativo para smartphone. Revista Transportes, [S. I.] v. 25, n. 2, p. 70-83, 2018. Disponível em: https://www.revistatransportes.org.br/anpet/article/view/1406/703. Acesso em: 27 de jan. 2020. 
BERNUCCI, L. B.; et al. Pavimentação asfáltica: formação básica para engenheiros. Rio de Janeiro: Petrobrás, 2010.

BISCONSINI, D. R. Avaliação da irregularidade longitudinal dos pavimentos com dados coletados por smartphones. 2016. Dissertação (Mestrado em Engenharia de Transportes) - Universidade de São Paulo, São Carlos, 2016. Disponível em: https://teses.usp.br/teses/disponiveis/18/18143/tde-18072016104745/publico/DANILORBISCONSINI.pdf. Acesso em: 20 abr. 2019.

BROWN, D.; LIU, W.; HENNING, T. F. P. Identifying pavement deterioration by enhancing the definition of road roughness. New Zealand: Trid, 2010.66p.

BUTTLAR, W. G.; ISLAM, S. Effect of pavement roughness on user costs. Transportation Research Record, v. 2285, n. 1, p. 47-55, 2012.

DEL ROSARIO, M. B; REDMOND, S. J; LOVELL N. H. Tracking the evolution of smartphone sensing for monitoring human movement. 2015. Sensors, [S. I], v. 15, n., 8, p. 18901-18933, 2015. Disponível em: http://dx.doi.org/10.3390/s15081901. Acesso em: 19 set. 2019.

DEPARTAMENTO NACIONAL DE ESTRADAS DE RODAGEM. Medição da irregularidade de superfície de pavimento com sistemas integradores IPR/USP e maysmeter: norma rodoviária: DNER-PRO 182/94. Rio de Janeiro: Ministério dos Transportes, 1994. Disponível em: http://ipr.dnit.gov.br/normas-e manuais/ normas/procedimento-pro/dner-pro182-94.pdf. Acesso em: 20 abr. 2019.

DUARTE, R. I. M. Análise comparativa da irregularidade longitudinal por faixas de tráfego na rodovia CE-401 obtida por aplicativo para smartphones. 2018. Trabalho de Conclusão de Curso (Bacharelado em Engenharia Civil) - Universidade Federal do Ceará, Fortaleza, 2018. Disponível em: http://repositorio. ufc.br/bitstream/riufc/40629/1/2018_tcc_rimduarte.pdf. Acesso em: 20 abr. 2019.

FORSLÖF, L.; JONES, H. Roadroid: continuous road condition monitoring with smart phones Journal of Civil Engineering and Architecture, [S. I.], v. 9, p. 486-496, 2015.

GILLESPIE, T. D.; Paterson, W. D. O.; Sayers, M. W. Guidelines for Conducting and Calibrating Road Roughness Measurements., [S. I.], 1986. 87p. (World Bank Technical Paper, 46).

GOOGLE. Google Earth Pro: versão 7.3.Disponível em: https://www.google.com.br/earth/download/gep/ agree.html. Acesso em: 15 abr. 2019.

KARAMIHAS, S. M.; SAYERS, M. W. The little book of profiling: basis information about measuring an interpreting road profiles. Michigan: University of Michigan. 1998.

LERCH, R. L. Previsão de irregularidade pós-recape em rodovias do RS: ajuste do modelo HDM4. 2002. 108 f. Dissertação (Mestrado em Engenharia) - Universidade Federal do Rio Grande do Sul. Porto Alegre, 2002. Disponível em: https://lume.ufrgs.br/bitstream/handle/10183/2981/000329912. pdf?sequence=1\&isAllowed=y. Acesso em: 20 mar. 2020.

PESQUISA CNT de rodovias 2018: relatório gerencial. 22. ed. Brasília: CNT, 2018. Disponível em: http:// cms.pesquisarodovias.cnt.org.br//Relatorio\%20Geral/Pesquisa\%20CNT\%20de\%20Rodovias\%202018\%20 -\%20web\%20-\%20baixa.pdf. Acesso em: 20 abr. 2019.

SCHLOTJES, M. R.; VISSER, A.; BENNET, C. Evaluation of a smartphone roughness meter. In: SOUTHERN AFRICAN TRANSPORT CONFERENCE, 33., 2014, Pretoria. Proceedings [...]. Petroria: CE Projects cc, 2014. p. 7-10. Disponível em: http://repository.up.ac.za/bitstream/handle/2263/45571/Visser_ Evaluation_2014.pdf?sequence=1\&isAllowed=y. Acesso em: 12 jun. 2019.

TOMIYAMA, K.; et al. Mobile profilometer for road surface monitoring by use of accelerometers. 7 . ed. Norfolk: SURF, 2012. 
WANG, W.; GUO, F. RoadLab: revamping road condition and road safety monitoring by crowdsourcing with Smartphone App. In: Proceedings [...]. TRANSPORTATION RESEARCH BOARD ANNUAL MEETING, 95, Washington, [s. n.] 2016, p. 1-15.

YU, J; CHOU, E; YAU, J. Development of speed-related ride quality thresholds using International Roughness Index. Transportation Research Record Journal of the Transportation Research Board, [S. I.], v. 1, p. 47-53, 1974.

\section{Sobre os autores}

José Wémenson Rabelo Chaves.

Graduando em Engenharia civil pela Faculdade Cisne Quixadá. Foi monitor nas disciplinas de Cálculo I e Geotécnica II. Técnico em Informática pela EEEP Professor Onélio Porto (2017).

\section{Renan Calixto Pereira}

Graduado em Engenheiro civil pela Faculdade Cisne de Quixadá (2019).

\section{Lucas Cavalcante de Almeida}

Mestre em Infraestruturas de Transportes pela Universidade Federal do Ceará - UFC (2018). Graduado em Engenharia civil pela Universidade de Fortaleza (2015) e em Engenharia de Teleinformática pela Universidade Federal do Ceará (2011).

\section{Francisco Heber Lacerda de Oliveira}

Doutor em Engenharia de Transportes pelo Programa de Pós-Graduação em Engenharia de Transportes da Universidade Federal do Ceará. Professor adjunto do Departamento de Engenharia de Transportes da Universidade Federal do Ceará. Affiliate Member in the American Society of Civil Engineers - ASCE. Tem experiência em planejamento do transporte aéreo, operação, manutenção e reabilitação de infraestruturas aeroportuárias, especialmente em pavimentos de pátios e de pistas de pouso e decolagem.

Recebido em: 10.04 .2020

Aceito em: 05.06.2020 